\title{
Electromagnetic structure of charmed baryons - extended to spin-3/2
}

\author{
K. U. Can*, M. Oka \\ Department of Physics, H-27, Tokyo Institute of Technology, \\ Meguro, Tokyo 152-8551 Japan \\ E-mail: utku.candth.phys.titech.ac.7p, ba@th.phys.titech.ac.jp \\ G. Erkol \\ Department of Natural and Mathematical Sciences, Faculty of Engineering, Ozyegin University, \\ Nisantepe Mah. Orman Sok. No:13, Alemdag 34794 Cekmekoy, Istanbul Turkey \\ E-mail: guray.erkoleozyeqin.edu.tr, bora.isildakeozvegin.edu.tr
}

\section{T. T. Takahashi}

Gunma National College of Technology,

Maebashi, Gunma 371-8530, Japan

E-mail: Etoruenat.qunma-ct.ac.jp

The electromagnetic form factors of the spin-3/2 $\Omega$ baryons, namely the $\Omega, \Omega_{c}^{*}, \Omega_{c c}^{*}$ and $\Omega_{c c c}$, are calculated in full QCD on $32^{3} \times 64$ PACS-CS lattices with a pion mass of $156(9) \mathrm{MeV}$. The electric charge radii and magnetic moments from the E0 and M1 multipole form factors are extracted. Results for the electric quadrupole form factor, E2, are also provided. Quark sector contributions are computed individually for each observable and then combined to obtain the baryon properties. Charm quark contributions are observed to be systematically smaller compared to the strange quark contributions in case of the charge radii and magnetic moments. E2 moments of the $\Omega_{c c}^{*}$ and $\Omega_{c c c}$ are estimated significantly enough to show that their electric charge distributions are deformed. Properties of the spin-1/2 counterparts of the $\Omega_{c}^{*}$ and $\Omega_{c c}^{*}$ baryons are also computed and a comparison is presented.

The 33rd International Symposium on Lattice Field Theory

14 - 18 July 2015

Kobe International Conference Center, Kobe, Japan*

\footnotetext{
* Speaker.
} 


\section{Introduction}

In this proceedings we report on our latest calculation of elastic electromagnetic form factors of charmed hadrons. This work extends our previous form factor calculation of spin-1/2 charmed baryons [W] to the spin-3/2 baryons composed of strange and charm quarks. Our aim is to broaden the perspective on heavy baryons by including the elastic electromagnetic form factors of the $J=\frac{3}{2}^{+}$strange-charmed baryons and examining the spin-dependence of the quark dynamics. We compute the electromagnetic form factors of the $\Omega, \Omega_{c}^{*}, \Omega_{c c}^{*}$ and $\Omega_{c c c}$ baryons as well as the $\Omega_{c}, \Omega_{c c}$ baryons with $J=\frac{1}{2}^{+}$. We extract the electric charge radii and the magnetic moments, and give a thorough comparison of both for the spin-3/2 and spin- $1 / 2$ sectors, which helps to improve our understanding of the nonperturbative structure of the strange-charmed baryons.

\section{Theoretical formulation and lattice setup}

Electromagnetic form factors of baryons can be calculated through their matrix elements of the electromagnetic vector current $j_{\mu}=\sum_{q} e_{q} \bar{q}(x) \gamma_{\mu} q(x)$, where $q$ runs over the quark content of the baryon in consideration. We refer the reader to Ref. [W] for the analytical expressions of spin-1/2 baryons. Here we only give the details for spin-3/2 baryons.

The electromagnetic transition matrix element for the spin- $3 / 2$ baryons can be written as

$$
\left\langle B_{\sigma}\left(p^{\prime}, s^{\prime}\right)\left|j_{\mu}\right| B_{\tau}(p, s)\right\rangle=\sqrt{\frac{M_{B}^{2}}{E E^{\prime}}} \bar{u}_{\sigma}\left(p^{\prime}, s^{\prime}\right) \mathscr{O}^{\sigma \mu \tau} u_{\tau}(p, s),
$$

where $p(s)$ and $p^{\prime}\left(s^{\prime}\right)$ denote the four momentum (spin) of the initial and final states, respectively. $M_{B}$ is the mass of the baryon, $E\left(E^{\prime}\right)$ is the energy of the incoming (outgoing) baryon state and $u_{\alpha}(p, s)$ is the baryon spinor in the Rarita-Schwinger formalism. The tensor in Eq. (ZD) can be written in a Lorentz-covariant form as [2]

$$
\mathscr{O}^{\sigma \mu \tau}=-g^{\sigma \tau}\left\{a_{1} \gamma^{\mu}+\frac{a_{2}}{2 M_{B}} P^{\mu}\right\}-\frac{q^{\sigma} q^{\tau}}{\left(2 M_{B}\right)^{2}}\left\{c_{1} \gamma^{\mu}+\frac{c_{2}}{2 M_{B}} P^{\mu}\right\}
$$

where $P=p+p^{\prime}$ and $q=p^{\prime}-p$. The multipole form factors we calculate in this work are defined in terms of the covariant vertex functions $a_{1}, a_{2}, c_{1}$ and $c_{2}$ as,

$$
\begin{aligned}
G_{E 0}\left(q^{2}\right) & =\left(1+\frac{2}{3} \tau\right)\left\{a_{1}+(1+\tau) a_{2}\right\}-\frac{1}{3} \tau(1+\tau)\left\{c_{1}+(1+\tau) c_{2}\right\}, \\
G_{E 2}\left(q^{2}\right) & =\left\{a_{1}+(1+\tau) a_{2}\right\}-\frac{1}{2}(1+\tau)\left\{c_{1}+(1+\tau) c_{2}\right\}, \\
G_{M 1}\left(q^{2}\right) & =\left(1+\frac{4}{3} \tau\right) a_{1}-\frac{2}{3} \tau(1+\tau) c_{1},
\end{aligned}
$$

with $\tau=-q^{2} /\left(2 M_{B}\right)^{2}$. These multipole form factors are referred to as electric-charge (E0), electric-quadrupole $(E 2)$ and magnetic-dipole $(M 1)$ multipole form factors.

We calculate the appropriate three-point correlation function on the lattice,

$$
\left\langle G_{\sigma \tau}^{B j^{\mu} B}\left(t_{2}, t_{1} ; \mathbf{p}^{\prime}, \mathbf{p} ; \Gamma\right)\right\rangle=-i \sum_{\mathbf{x}_{2}, \mathbf{x}_{1}} e^{-i \mathbf{p}^{\prime} \cdot \mathbf{x}_{2}} e^{i \mathbf{q} \cdot \mathbf{x}_{1}} \Gamma^{\alpha \alpha^{\prime}}\left\langle\operatorname{vac}\left|T\left[\eta_{\sigma}^{\alpha}\left(x_{2}\right) j_{\mu}\left(x_{1}\right) \bar{\eta}_{\tau}^{\alpha^{\prime}}(0)\right]\right| \operatorname{vac}\right\rangle,
$$


where the $\eta_{\mu}$ are the baryon interpolating fields chosen similarly to those of $\Delta$ baryon as $\eta_{\mu}(x)=$ $\frac{1}{\sqrt{3}} \varepsilon^{i j k}\left\{2\left[q_{1}^{T i}(x) C \gamma_{\mu} q_{2}^{j}(x)\right] q_{3}^{k}(x)+\left[q_{1}^{T i}(x) C \gamma_{\mu} q_{3}^{j}(x)\right] q_{2}^{k}(x)\right\} . i, j, k$ denote the color indices and $C=$ $\gamma_{4} \gamma_{2} . q_{1}, q_{2}, q_{3}$ are the quark flavors and taken as $\left(q_{1}, q_{2}, q_{3}\right)=\{(s, s, s),(s, s, c),(s, c, c),(c, c, c)\}$ for $\Omega, \Omega_{c}^{*}, \Omega_{c c}^{*}$ and $\Omega_{c c c}$ baryons, respectively. Spin projections with $\Gamma_{4}=\frac{1}{4}\left(1+\gamma_{4}\right)$ and $\Gamma_{i}=$ $\frac{1}{2}\left(\begin{array}{cc}\sigma_{i} & 0 \\ 0 & 0\end{array}\right)$ isolate the electric E0, E2 and magnetic M1 multipole form factors. In order to extract the matrix elements, we form a ratio of three-point to two-point functions which, in the large time limit $t_{2}-t_{1} \gg a$ and $t_{1} \gg a$, forms a time-independent ratio free from the unknown overlaps and exponential factors:

$$
R_{\sigma}{ }_{\tau}^{\mu}\left(t_{2}, t_{1} ; \mathbf{p}^{\prime}, \mathbf{p} ; \Gamma\right) \underset{t_{2}-t_{1} \gg a}{\stackrel{t_{1} \gg a}{\longrightarrow}}\left(\frac{E_{p}+M_{B}}{2 E_{p}}\right)^{1 / 2}\left(\frac{E_{p^{\prime}}+M_{B}}{2 E_{p^{\prime}}}\right)^{1 / 2} \Pi_{\sigma}{ }^{\mu} \tau\left(\mathbf{p}^{\prime}, \mathbf{p} ; \Gamma\right) .
$$

The multipole form factors can be extracted by using the following combinations of $\Pi_{\sigma}{ }^{\mu}{ }_{\tau}\left(\mathbf{p}^{\prime}, \mathbf{p} ; \Gamma\right)[$ [B]]:

$$
\begin{aligned}
G_{E 0}\left(q^{2}\right) & =\frac{1}{3}\left(\Pi_{11}^{4}\left(\mathbf{q}_{i}, 0 ; \Gamma_{4}\right)+\Pi_{22}^{4}\left(\mathbf{q}_{i}, 0 ; \Gamma_{4}\right)+\Pi_{33}^{4}\left(\mathbf{q}_{i}, 0 ; \Gamma_{4}\right)\right), \\
G_{E 2}\left(q^{2}\right) & =2 \frac{M(E+M)}{\left|\mathbf{q}_{i}\right|^{2}}\left(\Pi_{11}^{4}\left(\mathbf{q}_{i}, 0 ; \Gamma_{4}\right)+\Pi_{22}^{4}\left(\mathbf{q}_{i}, 0 ; \Gamma_{4}\right)-2 \Pi_{33}^{4}\left(\mathbf{q}_{i}, 0 ; \Gamma_{4}\right)\right), \\
G_{M 1}\left(q^{2}\right) & =-\frac{3}{5} \frac{E+M}{\left|\mathbf{q}_{1}\right|^{2}}\left(\Pi_{11}^{3}\left(\mathbf{q}_{1}, 0 ; \Gamma_{2}\right)+\Pi_{2}{ }_{2}\left(\mathbf{q}_{1}, 0 ; \Gamma_{2}\right)+\Pi_{3}{ }_{3}{ }_{3}\left(\mathbf{q}_{1}, 0 ; \Gamma_{2}\right)\right),
\end{aligned}
$$

where $i=1,2,3$ and $\mathbf{q}_{i}$ are the momentum vectors in three spatial directions.

We run our simulations on $32^{3} \times 64,2+1$-flavor configurations generated by the PACS-CS Collaboration [四] using the Clover fermion action and the Iwasaki gauge action. We use the gauge configurations at $\beta=1.90$ with the Clover coefficient $c_{S W}=1.715$, which give a lattice spacing of $a=0.0907(13) \mathrm{fm}(1 / a=2.176(31) \mathrm{GeV})$. The simulations are carried out on almost physical point ensembles with hopping parameter, $\kappa_{u, d}^{\text {sea }}, \kappa_{u, d}^{v a l}=0.13781$, which correspond to a pion mass of approximately $156 \mathrm{MeV}$. The strange quark mass is fixed to $\kappa_{\text {sea }}^{s}=0.13640$. In order to be consistent with the sea quarks we use the clover action for the $s$ valence quark propagators and we take $\kappa_{\text {sea }}^{s}=\kappa_{\text {val }}^{s}$.

For the charm quarks, we apply the Fermilab method [ []] in the form employed by the Fermilab Lattice and MILC Collaborations [ [6, U]. In this simplest form of the Fermilab method, the clover coefficients $c_{E}$ and $c_{B}$ in the action are set to the tadpole-improved value $1 / u_{0}^{3}$, where $u_{0}$ is the average link. Following the approach in Ref. [8]], we estimate $u_{0}$ to be the fourth root of the average plaquette. We tuned the charm-quark hopping parameter to $\kappa_{c}=0.1246$ by comparing the experimental spin-averaged static masses of charmonium and heavy-light mesons with our lattice results [四].

We make our simulations with the lowest allowed lattice momentum transfer $q=2 \pi /\left(N_{s} a\right)$, where $N_{s}$ is the spatial dimension of the lattice and $a$ is the lattice spacing. This corresponds to three-momentum squared value of $\mathbf{q}^{2}=0.183 \mathrm{GeV}^{2}$. In order to increase statistics, we insert all possible momentum components, namely $\left(\left|q_{x}\right|,\left|q_{y}\right|,\left|q_{z}\right|\right)=(-1,0,0),(0,-1,0),(0,0,-1)$, $(1,0,0),(0,1,0),(0,0,1)$. We also consider vector-current and spin projections along all spatial directions and take into account all Lorentz components of the Rarita-Schwinger field. By the wall method [0] that we employ, it is possible to extract all the components and simultaneously 
study all the hadrons we are considering without extra propagator inversions. The source interpolating fields are Gaussian smeared in a gauge-invariant manner and the source-sink separation is chosen to be 12 lattice units in the temporal direction. The smearing parameters for the $s$ quark are chosen so as to give a root-mean-square radius of $\left\langle r_{l}\right\rangle \sim 0.5 \mathrm{fm}$. As for the charm quark, we adjust the smearing parameters to obtain $\left\langle r_{c}\right\rangle=\left\langle r_{l}\right\rangle / 3$. All statistical errors are estimated by a single-elimination jackknife analysis. We calculate the connected diagrams only and consider the point-split lattice current, $j_{\mu}=1 / 2\left[\bar{q}(x+\mu) U_{\mu}^{\dagger}\left(1+\gamma_{\mu}\right) q(x)-\bar{q}(x) U_{\mu}\left(1-\gamma_{\mu}\right) q(x+\mu)\right]$, which is conserved by Wilson fermions.

\section{Results and Discussion}

Masses of the $\Omega, \Omega_{c}^{(*)}, \Omega_{c c}^{(*)}$ and $\Omega_{c c c}$ baryons are extracted from the shell-source/point-sink lattice two-point function by a simultaneous fit to all spatial Lorentz components. In units of $\mathrm{GeV}$ we find, $m_{\Omega_{c}}=2.783(13), m_{\Omega_{c c}}=3.747(10), m_{\Omega}=1.790(17), m_{\Omega_{c}^{*}}=2.837(18), m_{\Omega_{c c}^{*}}=$ $3.819(10)$ and $m_{\Omega_{c c c}}=4.769(6)$. For the $\Omega, \Omega_{c}$ and $\Omega_{c}^{*}$ baryons there is a discrepancy of around $100 \mathrm{MeV}$ compared to their experimental values, mainly due to the $\kappa_{s}^{\mathrm{val}}=0.13640$, which we choose same as the $\kappa_{s}^{s e a}$. For instance, $m_{\Omega}$ is in agreement with the $m_{\Omega}=1.772(7) \mathrm{GeV}$ reported by the PACS-CS Collaboration [四]. Mass of the $\Omega_{c c c}$ as obtained in our simulations agrees with

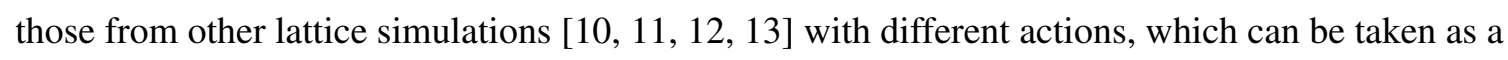
good indicator for the aptness of the charm-quark action we employ.

In our simulations we evaluate each quark sector separately and normalise to unit charge contributions. The baryon properties are then estimated by combining the quark contributions with their weights from respective quark numbers and electric charges as, $\langle\mathscr{O}\rangle=N_{s} e_{s}\left\langle\mathscr{O}_{s}\right\rangle+N_{c} e_{c}\left\langle\mathscr{O}_{c}\right\rangle$, where $\langle\mathscr{O}\rangle$ is the observable, $N_{q}$ is the number quarks inside the baryon having flavor $q$ and $e_{q}$ is the electric charge of the quark. Multipole form factor values are extracted by searching for plateau regions of the ratio given in Eq.(2.7). We refer the reader to Ref. [14]] for the illustration of ratios and the fit regions of the $E 0, M 1$ and $E 2$ form factors. Here we only quote the charge radii, magnetic moments and quadrupole moments.

Electric charge radii of the baryons are calculated by, $\left\langle r_{E}^{2}\right\rangle=-\left.6 \frac{d}{d Q^{2}} G_{E 0}\left(Q^{2}\right)\right|_{Q^{2}=0}$. We assume a dipole form Ansatz for the form factor, $G_{E 0}\left(Q^{2}\right)=\frac{G_{E 0}(0)}{\left(1+Q^{2} / \Lambda^{2}\right)^{2}}$, where $\Lambda$ is the dipole mass. Since we perform our simulations with a single value of the finite momentum transfer, we extract the charge radii using the expression, $\frac{\left\langle r_{E}^{2}\right\rangle}{G_{E 0}(0)}=\frac{12}{Q_{\min }^{2}}\left(\sqrt{\frac{G_{E 0}(0)}{G_{E 0}\left(Q_{m i n}^{2}\right)}}-1\right)$. Our numerical values for the electric charge radii are given in Table $\mathbb{W}$. Note that the quark sector contributions are for individual quarks of unit electric charge.

We observe that the $s$-quark contribution to the electric charge radii is almost independent of the quark-flavor composition of the baryon. The charge radii of both spin-1/2 and spin-3/2 baryons


quark contributions, the charge radii of all baryons are similar. The ratios of individual quark-flavor contributions in the spin-1/2 to that in the spin-3/2 sector are also shown in Fig. W. We observe that for the singly-charmed $\Omega_{c}$ baryon, $s$ - and $c$-quark charge distributions are insensitive to the spinflip of the $c$-quark whereas in the case of the doubly-charmed $\Omega_{c c}$ baryon the contributions of $s$ and $c$-quark to the charge radii increase. We find the electric charge radius of the $\Omega$ baryon to be $\left\langle r_{E}^{2}\right\rangle_{\Omega^{-}}=-0.326(21) \mathrm{fm}^{2}$ in quite good agreement with the previous lattice determinations [[15], []]. 


\begin{tabular}{c|cc|cccc}
\hline \hline & $\Omega_{c}$ & $\Omega_{c c}$ & $\Omega$ & $\Omega_{c}^{*}$ & $\Omega_{c c}^{*}$ & $\Omega_{c c c}$ \\
\hline \hline$\left\langle r_{E}^{2}\right\rangle_{s}\left[\mathrm{fm}^{2}\right]$ & $0.329(25)$ & $0.313(16)$ & $0.326(21)$ & $0.345(17)$ & $0.348(16)$ & - \\
$\left\langle r_{E}^{2}\right\rangle_{c}\left[\mathrm{fm}^{2}\right]$ & $0.064(11)$ & $0.073(4)$ & - & $0.062(5)$ & $0.078(3)$ & $0.084(3)$ \\
$\left\langle r_{E}^{2}\right\rangle\left[\mathrm{fm}^{2}\right]$ & $-0.177(18)$ & $0.026(4)$ & $-0.326(21)$ & $-0.189(12)$ & $-0.012(6)$ & $0.168(5)$ \\
\hline \hline
\end{tabular}

Table 1: Electric charge radii of the $\Omega, \Omega_{c}^{*}, \Omega_{c c}^{*}$ and $\Omega_{c c c}$. Results are given in $\mathrm{fm}^{2}$. Quark sector contributions are for single quark and normalised to unit charge. Total electric charge radius of the spin- $1 / 2 \Omega_{c}$ is estimated by combining quark sectors since its electric form factor vanishes due to its zero electric charge.

A comparison of baryon charge radii is made in Fig. W. In magnitude, $\Omega\left(\Omega_{c}^{*}\right)$ baryon has the largest electric charge radius among all (spin-3/2) baryons we study. Spin-1/2 (spin-3/2) $\Omega_{c}\left(\Omega_{c}^{*}\right)$ and $\Omega_{c c c}$ seem to have similar charge radii while the $\Omega_{c c}\left(\Omega_{c c}^{*}\right)$ baryon has almost a vanishing charge radius.
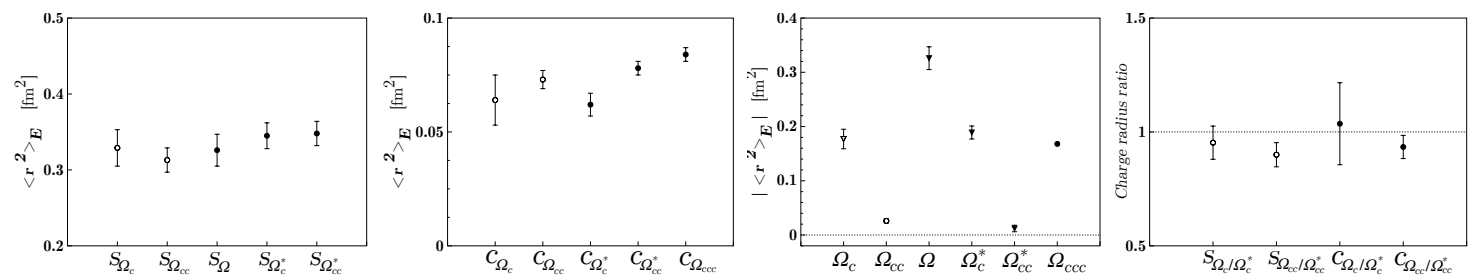

Figure 1: First three are: $s-$ and $c$-quark contributions to the electric charge radii and the total electric charge radii of the denoted baryons respectively. Last one is: the ratio of the quark contribution to the electric charge radii of the spin- $1 / 2 \Omega_{c}, \Omega_{c c}$ to spin-3/2 $\Omega, \Omega_{c}^{*}$ baryons. Absolute values are shown for a better comparison. Data points denoted by a triangle indicates a negative value. Empty symbols indicate the spin-1/2 sector.

We evaluate the magnetic dipole moment in units of nuclear magnetons as, $\mu_{B}=G_{M 1}(0) \frac{e}{2 m_{B}}=$ $G_{M 1}(0) \frac{m_{N}}{m_{B}} \mu_{N}$, where $m_{N}$ is the physical nucleon mass and $m_{B}$ is the baryon mass as obtained on the lattice. As in Ref. [16], we assume that the momentum-transfer dependence of the magnetic dipole form factor is same as the momentum dependence of the respective baryon's charge form factor. For instance, the scaling of $G_{M 1}$ is given by, $G_{M 1}^{s, c}(0)=G_{M 1}^{s, c}\left(q^{2}\right) \frac{G_{E}^{s, c}(0)}{G_{E}^{s c}\left(q^{2}\right)}$, where we consider the scaling of $s$ and $c$ quark sectors separately since each sector has a different scaling property. Quark sectors are then combined to construct $G_{M 1}(0)$.

Our numerical values for the magnetic moments are listed in Table $\square$, which are also illustrated in Fig. \. We find for spin-1/2 baryons that the contribution of a single quark to the magnetic moment significantly increases when it is doubly represented. The effect of the spin flip is seen as a sign change in case of the $\Omega_{c}$ and $\Omega_{c c}$ baryons. We illustrate the ratios of the quark-sector contributions to spin-1/2 and spin-3/2 baryons in Fig. $\square$ to understand the effect of the quark spin configurations on the quark magnetic moments better. In the case of spin-3/2 baryons, both the individual $s$-quark and $c$-quark contributions are enhanced. Ratios, $S_{\Omega_{c}^{\prime} \Omega_{c}^{*}}$, and $C_{\Omega_{c c}^{\prime} \Omega_{c c}^{*}}$, are consistent with each other like the singly strange and the singly charmed baryon ratios, suggesting that the difference between the spin-1/2 and the spin-3/2 baryons is almost independent of the quark flavour. We find the magnetic moment of the $\Omega^{-}$baryon to be $\mu_{\Omega^{-}}=-1.533 \pm 0.055 \mu_{N}$, which 


\begin{tabular}{l|cc|cccc}
\hline \hline & $\Omega_{c}$ & $\Omega_{c c}$ & $\Omega$ & $\Omega_{c}^{*}$ & $\Omega_{c c}^{*}$ & $\Omega_{c c c}$ \\
\hline \hline$\mu_{s}\left[\mu_{N}\right]$ & $0.979(47)$ & $-0.402(17)$ & $1.533(55)$ & $1.453(36)$ & $1.408(29)$ & - \\
$\mu_{c}\left[\mu_{N}\right]$ & $-0.092(6)$ & $0.216(3)$ & - & $0.358(8)$ & $0.352(4)$ & $0.338(2)$ \\
$\mu\left[\mu_{N}\right]$ & $-0.688(31)$ & $0.403(7)$ & $-1.533(55)$ & $-0.730(23)$ & $0.000(10)$ & $0.676(5)$ \\
\hline \hline
\end{tabular}

Table 2: Magnetic moments of the $\Omega, \Omega_{c}^{(*)}, \Omega_{c c}^{(*)}$ and $\Omega_{c c c}$. Results are given in units of nuclear magnetons, $\mu_{N}$. Quark sector contributions are for single quark and normalised to unit charge.

is smaller in magnitude than the experimental value, $\mu_{\Omega^{-}}^{\exp }=-2.02 \pm 0.05 \mu_{N}$ [미]. One of the reasons for this discrepancy can arise from the difference between our $m_{\Omega}=1.790(17)$ and the experimental value, $m_{\Omega}=1.673(29)$, which is around $7 \%$. Compared to the other lattice determinations that use the three-point function method, our value is slightly smaller (in magnitude) than the quenched result, $\mu_{\Omega^{-}}=-1.697 \pm 0.065 \mu_{N}$, of Boinepalli et.al [B]] and agrees with the Alexandrou et.al's extrapolated value, $\mu_{\Omega^{-}}=-1.875 \pm 0.399 \mu_{N}$ [ए5]] within one sigma error. In Ref.[18] magnetic moment of $\Omega$ has determined to be $\mu_{\Omega^{-}}=-1.93 \pm 0.08$, by a background field method on $m_{\pi}=366 \mathrm{MeV}$ lattices. Magnetic moments of $\Omega_{c}$ and $\Omega_{c}^{*}$ being similar to each other suggests that the spin flip of the charm quark has a small effect, in accord with heavy-quark spin symmetry expectations. From a quark-model approach one would expect the magnetic moment of $\Omega_{c}\left(\Omega_{c c}\right)$ to be similar in magnitude to that of $\Omega_{c}^{*}\left(\Omega_{c c}^{*}\right)$ 's. Such an expectation is consistent with our $\Omega_{c}$ findings, however, the magnetic moments of $\Omega_{c c}$ and $\Omega_{c c}^{*}$ differ drastically, the latter having a completely vanishing magnetic moment. The difference between the $\Omega_{c}$ and $\Omega_{c}^{*}$ is that the $c$-quark is anti-aligned with the ss component in $\Omega_{c}$ whereas it is aligned in $\Omega_{c}^{*}$. Combined with their electric charges, quark sectors add constructively for $\Omega_{c}$ and destructively for $\Omega_{c}^{*}$. These two different behaviours occur in such a balanced way that the magnetic moments of the $\Omega_{c}$ and $\Omega_{c}^{*}$ end up to be similar. In case of the doubly-charmed $\Omega_{c c}$ and $\Omega_{c c}^{*}$ however, the interplay between the electric charges and the number of quarks breaks the balance and lead to magnetic moments for $\Omega_{c c}$ and $\Omega_{c c}^{*}$ that differ significantly.
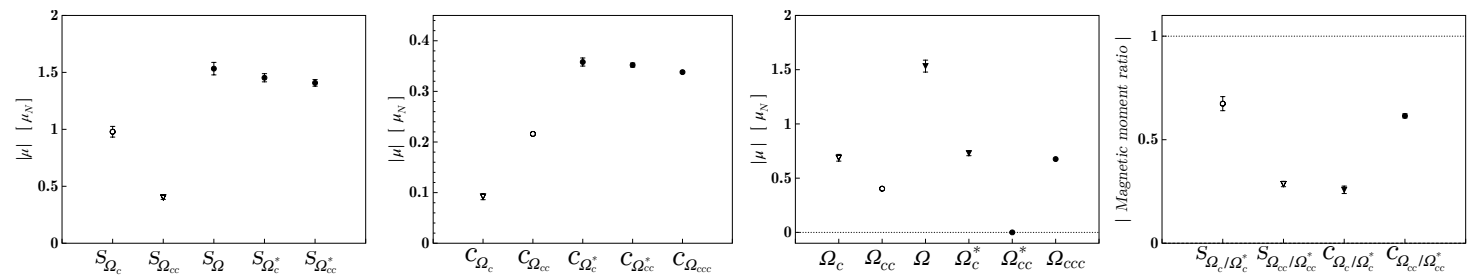

Figure 2: Same as Fig.1 but for magnetic moments.

The electric-quadrupole form factors of spin-3/2 baryons provide information about the deviation of the baryon shape from spherical symmetry. In the Breit frame, the quadrupole form factor and the electric charge distribution are related as [ㅍ] , $G_{E 2}(0)=M_{B}^{2} \int d^{3} r \bar{\psi}(r)\left(3 z^{2}-r^{2}\right) \psi(r)$, where $3 z^{2}-r$ is the standard operator used for quadrupole moments. A positively charged baryon has a prolate (oblate) charge distribution when quadrupole form factor is positive (negative). As in the case of the $E 0$ and $M 1$ form factors, we estimate the $E 2$ form factor by the plateau approach. We compute and extract the $s$ - and $c$-quark sector contributions individually. Unfortunately, low 
signal/noise ratio does not allow us to conclude about $\Omega$ and $\Omega_{c}^{*}$ baryons. In the case of the heavier $\Omega_{c c}^{*}$ and $\Omega_{c c c}$ baryons, however, we find $E_{2}\left(Q^{2}\right)^{\Omega_{c c}^{*}}=-0.310(128)$ and $E_{2}\left(Q^{2}\right)^{\Omega_{c c c}}=-0.273(76)$ indicating that their charge distributions deform to an oblate shape.

\section{Summary}

We have calculated the electromagnetic form factors of the $\Omega, \Omega_{c}^{*}, \Omega_{c c}^{*}$ and $\Omega_{c c c}$ baryons at the lowest allowed three-momentum value $\left(\mathbf{q}^{2}=0.183 \mathrm{GeV}^{2}\right)$ and extracted their electric charge radii, magnetic moments and quadrupole moments on almost physical point gauge configurations. Same quantities for the $\Omega_{c}$ and $\Omega_{c c}$ baryons are also computed. For each observable we have identified the quark sector contributions and gave a comparison between the quark sectors and spin-1/2 and spin-3/2 sectors. In addition, baryon properties are constructed from the individual quark sectors contributions.

Acknowledgments: Part of numerical calculations in this work were performed on National Center for High Performance Computing of Turkey (Istanbul Technical University) under project number 10462009. The unquenched gauge configurations employed in our analysis were generated by PACS-CS collaboration [四]. We used a modified version of Chroma software system [20] along with QUDA [2], 22]. This work is supported in part by The Scientific and Technological Research Council of Turkey (TUBITAK) under project number 114F261 and in part by KAKENHI under Contract Nos. 25247036 and 24250294. This work is also supported by the Research Abroad and Invitational Program for the Promotion of International Joint Research, Category (C) and the International Physics Leadership Program at Tokyo Tech. G. Erkol would like to thank M. Oka and Tokyo Institute of Technology, where part of this work was done, for the hospitality they provided.

\section{References}

[1] K. U. Can, G. Erkol, B. Isildak, M. Oka and T. T. Takahashi, JHEP 1405, 125 (2014)

[2] S. Nozawa and D. B. Leinweber, Phys. Rev. D42, 3567-3571 (1990)

[3] S. Boinepalli, D. B. Leinweber, P. J. Moran, A. G. Williams, J. M. Zanotti and J. B. Zhang, Phys.Rev. D80, 054505 (2009)

[4] S. Aoki et al. [PACS-CS Collaboration], Phys. Rev. D79, 034503 (2009).

[5] A. X. El-Khadra, A. S. Kronfeld and P. B. Mackenzie, Phys. Rev. D55, 3933-3957 (1997)

[6] T. Burch, C. DeTar, M. Di Pierro, A.X. El-Khadra, E. D. Freeland et al., Phys. Rev. D81, 034508 (2010)

[7] C. Bernard et al, Phys. Rev. D83, 034503 (2011)

[8] D. Mohler and R. M. Woloshyn, Phys. Rev. D83, 034503 (2011)

[9] K. U. Can, G. Erkol, M. Oka, A. Ozpineci and T. T Takahashi,Phys. Lett. B719, 103 (2013).

[10] Y. Namekawa et al. [PACS-CS Collaboration], Phys. Rev. D87, 094512 (2013).

[11] C. Alexandrou, V. Drach, K. Jansen, C. Kallidonis and G. Koutsou, Phys. Rev. D90, 074501 (2014)

[12] R. A. Briceno, H-W. Lin and D. R. Bolton, Phys. Rev. D86, 094504 (2012)

[13] Z. S. Brown, W. Detmold, S. Meinel and K. Orginos, Phys. Rev. D90, 094507 (2014)

[14] K. U. Can, G. Erkol, M. Oka and T. T. Takahashi, (2015), [arXiv: 1508.03048].

[15] C. Alexandrou, T. Korzec, G. Koutsou, J. W. Negele and Y. Proestos, Phys. Rev. D82, 034504 (2010).

[16] D. B. Leinweber, T. Draper and R. M. Woloshyn, Phys. Rev. D48, 2230-2249 (1993).

[17] K.A. Olive and Particle Data Group, Chinese Physics C 38, 090001 (2014).

[18] C. Aubin, K. Orginos, V. Pascalutsa and M. Vanderhaeghen, Phys. Rev. D79, 051502 (2009).

[19] D. B. Leinweber, T. Draper and R. M. Woloshyn, Phys. Rev. D46, 3067-3085 (1992).

[20] R. G. Edwards and B. Joo [SciDAC Collaboration, LHPC Collaboration, UKQCD Collaboration], Nucl.Phys.Proc.Suppl. 140, 832 (2005).

[21] R. Babich, M. A. Clark, M.A. B. Joo, G. Shi, R. C. Brower et al., (2011), [arXiv: 1109.2935$].$

[22] M. A. Clark, R. Babich, K. Barros, R. C. Brower and C. Rebbi, Comput. Phys. Commun. 181, 1517-1528 (2010). 